\title{
Completely Integrable Generalized C. Neumann Systems on Several Symplectic Submanifolds ${ }^{\star}$
}

\author{
Zhijun QIAO \\ Institute of Mathematics, Fudan University, Shanghai 200433, and \\ Department of Mathematics, Liaoning University, Shenyang 110036 \\ Submitted by W. FUSHCHYCH \\ Received November 14, 1995
}

Abstract

New completely integrable generalized C. Neumann systems on several symplectic submanifolds are presented, and the relations between the generalized C. Neumann systems and the spectral problems are further discussed in this paper. In particular, a new eigenvalue problem is proposed in Part 3.3.

\section{Introduction}

Owing to the excellent works of Cao [1,2], the nonlinearization method about a spectral problem or Lax pair plays a very important part in finding out new completely integrable systems as many as possible. It is known that the nonlinearized systems of some spectral problems or Lax pairs depend on some certain constraints between the potentials and the eigenfunctions. These constraints contain the Bargmann constraint [2], the C. Neumann constraint [2], and the symmetry constraint [3]. The Bargmann constraints are developed to construct completely integrable systems in the Liouville sense by several authors $[4$, 5]. The symmetry constraints reveal the relations between higher dimensional integrable systems and lower dimensional integrable systems and give a direct method to construct solutions of higher dimensional integrable systems by solving lower dimensional integrable systems [6, 7]. Many Bargmann constraints and symmetry constraints have been studied, and the former reduce $(1+1)$-dimensional integrable systems to finite dimensional completely integrable systems (see ref. $[2,4,5])$ and the latter reduce $(1+2)$-dimensional integrable systems to $(1+1)$-dimensional integrable systems (see ref. $[3,6,7])$. Compared with the above two such constraints, few C. Neumann constraints and C. Neumann systems are established. Among already known C. Neumann and C. Neumann systems, there are ones associated with the KdV hierarchy [2], Jaulent-Miodek hierarchy [2]. Tu Guizhang hierarchy [2], coupled KdV hierarchy [4], the hierarchy $[8,15]$ of evolution equations related with the novel, spectral parameter dependent Schrödinger equation $\lambda \Phi_{x x}+\sum_{i=1}^{m-1} \lambda^{i} u_{i} \Phi=\lambda^{m} \Phi$, WKI hierarchy [9] and Levi hierarchy [10]. The cause of a small number of C. Neumann constraints and C. Neumann systems is maybe in the complicated difficult calculations and some modifications. Especially, some results are sometimes guessed for the C. Neumann system.

Copyright (C) 1997 by Mathematical Ukraina Publisher. All rights of reproduction in any form reserved.

${ }^{\star}$ The Project (\# 19501019) Supported by Natural Foundation of China. 
In this paper, we would like to present some new completely integrable generalized C. Neumann systems on several symplectic submanifolds according to the Moser constraint method [11]. Simultaneously, we shall see that such symplectic submanifolds are exactly generated by the C. Neumann constraint conditions [2] between the potential and eigenfunctions of several spectral problems. Particulary, a new eigenvalue problem is proposed in Part 3.3.

For the convenience of use, in the following we give some fundamental notations and symbols. Let $\lambda_{j},(1 \leq j \leq N)$ be $N$ different real (or complex) constants, $\lambda_{1}<\lambda_{2}<\cdots<$ $\lambda_{n}, \Lambda=\operatorname{diag}\left(\lambda_{1}, \ldots, \lambda_{n}\right)$. For $p=\left(p_{1}, \ldots, p_{n}\right)^{T}, q=\left(q_{1}, \ldots, q_{n}\right)^{T}$, write the coordinate space $R^{2 N}=\left\{(p, q)^{T}\right\}$ and denote by the differential 2 -form $\omega^{2}=d p \wedge d q$ the symplectic structure in $R^{2 N} .<\cdot, \cdot>$ stands for the standard inner product in $R^{2 N}$. Then, for two arbitrary functions $F, G$ in the symplectic manifold $\left(R^{2 N}, \omega^{2}=d p \wedge d q\right)$, define the Poisson bracket of them as follows [12]

$$
(F, G)=\sum_{j=1}^{n}\left(\frac{\partial F}{\partial q_{j}} \frac{\partial G}{\partial p_{j}}-\frac{\partial F}{\partial p_{j}} \frac{\partial G}{\partial q_{j}}\right)=\left\langle\frac{\partial F}{\partial q}, \frac{\partial G}{\partial p}\right\rangle-\left\langle\frac{\partial F}{\partial p}, \frac{\partial G}{\partial q}\right\rangle .
$$

$f, G$ are called involutive, if $(F, G)=0$.

\section{C. Neumann system of the Zakharov-Shabat eigenvalue problem}

We have already known the Hamiltonian system $\left(R^{2 N}, d p \wedge d q, H\right)$ i.e., the nonlinearized system of the famous Zakharov-Shabat eigenvalue problem under the so-called Bargmann constraint [1]

$$
(H):\left\{\begin{array}{l}
q_{x}=i \Lambda q+<q, q>p=\frac{\partial H}{\partial p} \\
p_{x}=-i \Lambda p-<p, p>q=-\frac{\partial H}{\partial q}
\end{array}\right.
$$

is completely integrable in the Liouville sense and has the Hamiltonian

$$
H=i<\Lambda q, p>+\frac{1}{2}<p, p><q, q>
$$

and the conserved integrals involute in pairs $F_{m}$ :

$$
F_{m}=i<\Lambda^{m} q, p>+\frac{1}{2} \sum_{i+j=m-1}\left|\begin{array}{cc}
<\Lambda^{i} q, q> & <\Lambda^{i} q, p> \\
<\Lambda^{j} p, q> & <\Lambda^{j} p, p>
\end{array}\right|, \quad m=0,1,2, \ldots
$$

Now, we restrict the Hamiltonian system $(H)$ onto the symplectic submanifold defined by

$$
M^{2 N-2}=\left\{(p, q) \in R^{2 N} \mid F \equiv \frac{1}{2}(<q, q>-1)=0, G \equiv \frac{1}{2}(<p, p>-1)=0\right\} .
$$

On $M^{2 N-2}$, we consider the Hamiltonian $H^{*}=H-\lambda F-\mu G$, where $\lambda, \mu$ are the two Lagrange multipliers which are determined by the tangent conditions $\left(H^{*}, F\right)=0$, 
$\left(H^{*}, G\right)=0$. It is easy to calculate

$$
\begin{aligned}
& (f, G)=<p, q>,(H, F)=-i<\Lambda q, q>-<p, q>,(H, G)=i<\Lambda p, p>+<p, q> \\
& \lambda=\left.\frac{(H, G)}{(F, G)}\right|_{M^{2 N-2}}=i \frac{<\Lambda p, p>}{<p, q>}+1, \quad \mu=\left.\frac{(H, F)}{(G, F)}\right|_{M^{2 N-2}}=i \frac{<\Lambda q, q>}{<p, q>}+1 .
\end{aligned}
$$

Thus, we have

Proposition 1 The Hamiltonian system $\left(M^{2 N-2},\left.d p \wedge d p\right|_{M^{2 N-2}}, H^{*}\right)$ on the symplectic submanifold $M^{2 N-2}$

$$
\left(H^{*}\right):\left\{\begin{array}{l}
q_{x}=\frac{\partial H^{*}}{\partial p}=i \Lambda q-i \frac{<\Lambda q, q>}{<p, q>} p \\
p_{x}=-\frac{\partial H^{*}}{\partial p}=-i \Lambda p+i \frac{<\Lambda p, p>}{<p, q>} q
\end{array}\right.
$$

is completely integrable and its involutive system of conserved integrals $F_{m}^{*}$ is given by

$$
F_{m}^{*}=F_{m}-\lambda_{m} F-\mu_{m} G
$$

with

$$
\lambda_{m}=\left.\frac{\left(F_{m}, G\right)}{(F, G)}\right|_{M^{2 N-2}}=i \frac{<\Lambda^{m} p, p>}{<p, q>}, \quad \mu_{m}=\left.\frac{\left(F_{m}, F\right)}{(G, F)}\right|_{M^{2 N-2}}=i \frac{<\Lambda^{m} q, q>}{<p, q>} .
$$

Proof. By the tangent conditions $\left(F_{m}^{*}, F\right)=0,\left(F_{m}^{*}, G\right)=0$, the two Lagrange multipliers $\lambda_{m}$ and $\mu_{m}$ in (6) can be easily obtained. Through a lengthy careful calculations, we can know the Poisson bracket $\left(F_{m}^{*}, F_{n}^{*}\right)=0, \forall m, n \in Z^{+}$. Thus, the Hamiltonian system (5) is completely integrable in the Liouville sense.

In (5), set

$$
u=-i \frac{<\Lambda q, q>}{<p, q>}, \quad v=i \frac{<\Lambda p, p>}{<p, q>} .
$$

Then (5) becomes

$$
\left\{\begin{array}{l}
q_{j, x}=i \lambda_{j} q_{j}+u p_{j} \\
p_{j, x}=v q_{j}-i \lambda_{j} p_{j}
\end{array} \quad j=1,2, \ldots, N\right.
$$

which is none other than the well known Zakharov-Shabat eigenvalue problem

$$
y_{x}=\left(\begin{array}{rr}
i \lambda & u \\
v & -i \lambda
\end{array}\right) y, \quad i^{2}=-1
$$

with $y=\left(q_{j}, p_{j}\right)^{T}, \lambda=\lambda_{j}$.

We easily get the spectral gradient $\nabla \lambda_{j}$ of the spectral parameter $\lambda_{j}$ with respect to the potentials $u$ and $v$

$$
\nabla \lambda_{j}=\left(\begin{array}{c}
\delta \lambda_{j} / \delta u \\
\delta \lambda_{j} / \delta v
\end{array}\right)=\left(\begin{array}{r}
p_{j}^{2} \\
-q_{j}^{2}
\end{array}\right) .
$$


Consider the so-called C. Neumann constraint:

$$
(1,-1)^{T}=\sum_{j=1}^{N} \nabla \lambda_{j}
$$

which is equivalent to $\langle p, p\rangle=1,\langle q, q\rangle=1$, i.e. (11) exactly yields the symplectic submanifold $M^{2 N-2}$ in $R^{2 N}$. By making the $x$-derivative in Eq. (10) and using (8), we immediately know that (11) implies (7).

Definition The nonlinearized system of the ZS eigenvalue problem (9) under the C. Neumann constraint conditions (11) and (7) is called generalized C. Neumann system of the ZS eigenvalue problem (9).

By this definition, we have

Proposition 2 The symplectic submanifold $\left(M^{2 N-2},\left.d p \wedge d q\right|_{M^{2 N-2}}\right)$ defined by (4) in the symplectic space $\left(R^{2 N-2}, d p \wedge d q\right)$ is generated by the C. Neumann constraint (11) of ZS eigenvalue problem (9), and the completely integrable Hamiltonian system (5) on $M^{2 N-2}$ is exactly the generalized $C$. Neumann system of the $Z S$ eigenvalue problem (9).

Analogously to the above ZS case, other generalized C. Neumann systems connected with some spectral problems can be produced through introducing the concrete C. Neumann constraint (its form is like to (11)). Here, other three such examples are discussed below.

\section{Other three completely integrable generalized C. Neumann systems}

3.1. It is known that the completely integrable Hamiltonian system $\left(R^{2 N}, d p \wedge d q, H\right)$

$$
\begin{aligned}
& (H):\left\{\begin{array}{l}
q_{x}=\frac{\partial H}{\partial p}=-\Lambda q-<q, q>\Lambda p \\
p_{x}=-\frac{\partial H}{\partial q}=\Lambda p+<\Lambda p, p>q
\end{array}\right. \\
& H=-<\Lambda q, p>-\frac{1}{2}<q, q><\Lambda p, p>
\end{aligned}
$$

has the involutive system [2]

$$
\begin{gathered}
F_{m}=\frac{1}{2}<p, p><\Lambda^{m} q, q>-<\Lambda^{m} q, p>-<q, p><\Lambda^{m} p, q>- \\
\frac{1}{2} \sum_{j=0}^{m}\left|\begin{array}{cc}
<\Lambda^{j} q, q> & <\Lambda^{j} q, p> \\
<\Lambda^{m-j} p, q> & <\Lambda^{m-j} p, p>
\end{array}\right|, \quad m=0,1,2, \ldots
\end{gathered}
$$

here $H=F_{1}$.

Let us constraint the Hamiltonian $H$ defined by $(12)$ onto the $(2 N-2)$-dimensional symplectic submanifold $M^{2 N-2}$

$$
M^{2 N-2}=\left\{(p, q) \in R^{2 N} \mid F \equiv \frac{1}{2}(<\Lambda p, p>-1)=0, G \equiv \frac{1}{2}(<q, q>-1)=0\right\} .
$$


Then, like the calculation procedure in Sec. 2, we should choose

$$
\begin{aligned}
H^{*} & =H-\lambda F-\mu G, \\
\lambda & =\left.\frac{(H, G)}{(F, G)}\right|_{M^{2 N-2}}=-\frac{<\Lambda q, q>+<\Lambda p, q>}{<\Lambda p, q>}, \\
\mu & =\left.\frac{(H, F)}{(G, F)}\right|_{M^{2 N-2}}=-\frac{<\Lambda^{2} p, p>+<\Lambda p, q>}{<\Lambda p, q>}
\end{aligned}
$$

as the Hamiltonian function on $M^{2 N-2}$. Hence, we obtain

Proposition 3 The Hamiltonian function system $\left(H^{*}\right)$ on $M^{2 N-2}$

$$
\left(H^{*}\right):\left\{\begin{array}{l}
q_{x}=-\Lambda q+\frac{<\Lambda q, q>}{<\Lambda q, p>} \Lambda p=\frac{\partial H^{*}}{\partial p} \\
p_{x}=\Lambda p-\frac{<\Lambda^{2} p, p>}{<\Lambda q, p>} q=-\frac{\partial H^{*}}{\partial p}
\end{array}\right.
$$

is integrable and its involute function system $F_{m}^{*}$ is determined by

$$
F_{m}^{*}=F_{m}-\lambda_{m} F-\mu_{m} G
$$

with the Lagrange multipliers

$$
\begin{aligned}
& \lambda_{m}=\left.\frac{\left(F_{m}, G\right)}{(F, G)}\right|_{M^{2 N-2}}=-\frac{<\Lambda^{m} q, q>+<\Lambda^{m} q, p>}{<\Lambda p, q>}, \\
& \mu_{m}=\left.\frac{\left(F_{m}, F\right)}{(G, F)}\right|_{M^{2 N-2}}=-\frac{<\Lambda^{m+1} p, p>+<\Lambda^{m} q, p>}{<\Lambda p, q>} .
\end{aligned}
$$

In (17), order

$$
u=\frac{<\Lambda q, q>}{<\Lambda q, p>}, \quad v=-\frac{<\Lambda^{2} p, p>}{<\Lambda q, p>} .
$$

Then, (17) is exactly produced by the eigenvalue problem [13]

$$
y_{x}=\left(\begin{array}{rr}
-\lambda & \lambda u \\
v & \lambda
\end{array}\right) y
$$

with $y=\left(q_{j}, p_{j}\right)^{T}, \lambda=\lambda_{j}$, and (20) is deduced none other than the C. Neumann constraint

$$
(1,-1)^{T}=\sum_{j=1}^{N} \nabla \lambda_{j}, \quad \nabla \lambda_{j}=\left(\begin{array}{c}
\delta \lambda_{j} / \delta u \\
\delta \lambda_{j} / \delta v
\end{array}\right)=\left(\begin{array}{c}
\lambda_{j} p_{j}^{2} \\
-q_{j}^{2}
\end{array}\right)
$$

through the $x$-derivative of $(22)$.

Proposition 4 The symplectic submanifold $\left(M^{2 N-2},\left.d p \wedge d q\right|_{M^{2 N-2}}\right)$ defined by (14) in the symplectic space $\left(R^{2 N-2}, d p \wedge d q\right)$ is generated by the C. Neumann constraint (22), and the completely integrable Hamiltonian system (17) is actually the generalized C. Neumann system of the eigenvalue problem (21). 
3.2. The integrable Hamiltonian system $\left(C^{2 N}, d p \wedge d q, H\right)$

$$
(H):\left\{\begin{array}{l}
q_{x}=\frac{\partial H}{\partial p} \\
p_{x}=-\frac{\partial H}{\partial q}
\end{array} \quad H=-\frac{1}{2}<\Lambda q, q>+\frac{1}{2}<p, q>^{2}+\frac{1}{2}<p, p>\right.
$$

possesses the involutive system of conserved integrals $F_{m}\left(F_{0}=H\right)$

$$
\begin{gathered}
F_{m}=-\frac{1}{2}<\Lambda^{m+1} q, q>+\frac{1}{2}<\Lambda^{m} p, p>+\frac{1}{2}<q, q><\Lambda^{m} p, p>- \\
\frac{1}{2} \sum_{i+j=m}\left|\begin{array}{cc}
<\Lambda^{i} q, q> & <\Lambda^{i} q, p> \\
\left\langle\Lambda^{j} p, q>\right. & <\Lambda^{j} p, p>
\end{array}\right|, \quad m=0,1,2, \ldots
\end{gathered}
$$

Firstly, let us constrain the Hamiltonian $H$ onto the symplectic submanifold $M^{2 N-4}$

$$
\begin{aligned}
M^{2 N-4} & =\left\{(p, q) \in C^{2 N} \mid F^{1} \equiv \frac{1}{2}\left(<\Lambda^{-1} p, p>-1\right)=0, G^{1} \equiv<\Lambda^{-1} p, q>=0,\right. \\
F^{2} & \left.\equiv \frac{1}{2}\left(<\Lambda^{-1} p, p>+<q, q>\right)=0, G^{2} \equiv\left(<\Lambda^{-2} p, p>-<\Lambda^{-1} q, q>\right)=0\right\} .
\end{aligned}
$$

Then the Hamiltonian $H^{*}$ on $M^{2 n-4}$ should be set as

$$
H^{*}=H-\lambda^{1} F^{1}-\mu^{1} G^{1}-\lambda^{2} F^{2}-\mu^{2} G^{2},
$$

where the Lagrange multipliers $\lambda^{1}, \lambda^{2}, \mu^{1}, \mu^{2}$ determined by the tangent conditions $\left(H^{*}, F^{1}\right)=0,\left(H^{*}, F^{2}\right)=0,\left(H^{*}, G^{1}\right)=0,\left(H^{*}, G^{2}\right)=0$ are

$$
\begin{aligned}
& \lambda^{1}=\mu^{1}=\mu^{2}=0, \\
& \lambda^{2}=\left.\frac{\left(H, G^{2}\right)}{\left(F^{2}, G^{2}\right)}\right|_{M^{2 N-4}}=\frac{<q, p>\left(<\Lambda^{-1} q, q>+<\Lambda^{-2} p, p>\right)}{2<\Lambda^{-2} q, p>} .
\end{aligned}
$$

Proposition 5 The Hamiltonian system $\left(H^{*}\right)$ on $M^{2 N-4}$ defined by (25)

$\left(H^{*}\right):\left\{\begin{array}{l}q_{x}=\frac{\partial H^{*}}{\partial p}=p+<p, q>q-\frac{<p, q>\left(<\Lambda^{-1} q, q>+<\Lambda^{-2} p, p>\right)}{2<\Lambda^{-2} q, p>} \Lambda^{-1} p \\ p_{x}=-\frac{\partial H^{*}}{\partial p}=\Lambda q-<p, q>p+\frac{<p, q>\left(<\Lambda^{-1} q, q>+<\Lambda^{-2} p, p>\right)}{2<\Lambda^{-2} q, p>} q\end{array}\right.$

is completely integrable, and its involutive system $F_{m}^{*}$ is

$$
F_{m}^{*}=F_{m}-\lambda_{m}^{1} F^{1}-\mu_{m}^{1} G^{1}-\lambda_{m}^{2} F^{2}-\mu_{m}^{2} G^{2}
$$

with

$$
\begin{aligned}
& \lambda_{m}^{1}=\mu_{m}^{1}=\mu_{m}^{2}=0, \\
& \lambda_{m}^{2}=\left.\frac{\left(F_{m}, G^{2}\right)}{\left(F^{2}, G^{2}\right)}\right|_{M^{2 N-4}}=\frac{<\Lambda^{m} p, q>\left(<\Lambda^{-1} q, q>+<\Lambda^{-2} p, p>\right)}{2<\Lambda^{-2} p, q>} .
\end{aligned}
$$


In (28), set

$$
v=<p, q>, \quad u=-\frac{<p, q>\left(<\Lambda^{-1} q, q>+<\Lambda^{-2} p, p>\right)}{2<\Lambda^{-2} p, q>} .
$$

Then the Hamiltonian system $\left(H^{*}\right)$ becomes

$$
\left\{\begin{array}{l}
q_{x}=p+v q+u \Lambda^{-1} p \\
p_{x}=\Lambda q-u q-v p
\end{array}\right.
$$

which is exactly one eigenvalue problem studied by $\mathrm{Hu}[14]$

$$
y_{x}=\left(\begin{array}{rr}
v & 1+\lambda^{-1} u \\
\lambda-u & -v
\end{array}\right) y
$$

with $y=\left(q_{j}, p_{j}\right)^{T}, \lambda=\lambda_{j}$.

Proposition 6 The symplectic submanifold $\left(M^{2 N-4},\left.d p \wedge d q\right|_{M^{2 N-4}}\right)$ defined by (25) in the symplectic space $\left(R^{2 N-2}, d p \wedge d q\right)$ is generated by the $C$. Neumann constraint of the eigenvalue problem (33), and the completely integrable Hamiltonian system (28) is actually the generalized C. Neumann system of the eigenvalue problem (33).

Secondly, let us constrain the Hamiltonian $H$ onto the symplectic submanifold $M^{2 N-4}$

$M^{2 N-4}=\left\{(p, q) \in C^{2 N} \mid F^{1} \equiv \frac{1}{2}\left(<\Lambda^{-1} p, p>-1\right)=0, G^{1} \equiv<\Lambda^{-1} p, q>=0\right.$,

$\left.F^{2} \equiv \frac{1}{2}\left(<\Lambda^{-1} p, p>-<q, q>-2\right)=0, G^{2} \equiv \frac{1}{2}\left(<\Lambda^{-2} p, p>+<\Lambda^{-1} q, q>\right)=0\right\}$.

Proposition 7 The Hamiltonian system $\left(H^{*}\right)$ on $M^{2 N-4}$ defined by (34)

$\left(H^{*}\right):\left\{\begin{array}{l}q_{x}=\frac{\partial H^{*}}{\partial p}=p+<p, q>q+\frac{<p, q>\left(<\Lambda^{-2} p, p>-<\Lambda^{-1} q, q>\right)}{2<\Lambda^{-2} p, q>} \Lambda^{-1} p \\ p_{x}=-\frac{\partial H^{*}}{\partial p}=\Lambda q-<p, q>p+\frac{<p, q>\left(<\Lambda^{-2} q, q>-<\Lambda^{-1} q, q>\right)}{2<\Lambda^{-2} p, q>} q\end{array}\right.$

with

$$
H^{*}=H-\lambda^{2} F^{2}, \lambda^{2}=\left.\frac{\left(H, G^{2}\right)}{\left(F^{2}, G^{2}\right)}\right|_{M^{2 N-4}}=-\frac{<p, q>\left(<\Lambda^{-2} p, p>-<\Lambda^{-1} q, q>\right)}{2<\Lambda^{-2} p, q>},
$$

is completely integrable, and has the involute system $F_{m}^{*}$ :

$$
F_{m}^{*}=F_{m}-\lambda_{m}^{2} F^{2}, \lambda_{m}^{2}=\left.\frac{\left(F_{m}, G^{2}\right)}{\left(F^{2}, G^{2}\right)}\right|_{M^{2 N-4}}=-\frac{<\Lambda^{m} p, q>\left(<\Lambda^{-2} p, p>-<\Lambda^{-1} q, q>\right)}{2<\Lambda^{-2} p, q>} .
$$

In (35), set

$$
u=<p, q>, \quad v=-\frac{<p, q>\left(<\Lambda^{-2} p, p>-<\Lambda^{-1} q, q>\right)}{2<\Lambda^{-2} p, q>}
$$

then (35) is exactly changed as another eigenvalue problem studied by $\mathrm{Hu}$ [14]

$$
y_{x}=\left(\begin{array}{rr}
u & 1+\lambda^{-1} v \\
\lambda+v & -u
\end{array}\right) y
$$

with $y=\left(q_{j}, p_{j}\right)^{T}, \lambda=\lambda_{j}$. 
Proposition 8 The symplectic submanifold $\left(M^{2 N-4},\left.d p \wedge d q\right|_{M^{2 N-4}}\right)$ defined by (34) in the symplectic space $\left(R^{2 N-2}, d p \wedge d q\right)$ is generated by the $C$. Neumann constraint of the eigenvalue problem (38), and the completely integrable Hamiltonian system (35) is actually the generalized C. Neumann system of the eigenvalue problem (38).

3.3. It isn't difficult to prove that the Hamiltonian system $\left(R^{2 N}, d p \wedge d q, H\right)$

$$
\begin{aligned}
& (H):\left\{\begin{array}{l}
q_{x}=\frac{\partial H}{\partial p}=\Lambda p-<p, q>p \\
p_{x}=-\frac{\partial H}{\partial q}=\Lambda q-2<q, q>q+<p, q>p
\end{array}\right. \\
& H=\frac{1}{2}(<\Lambda p, p>-<\Lambda q, q>)+\frac{1}{2}<q, q>^{2}-\frac{1}{2}<p, q>^{2}
\end{aligned}
$$

is completely integrable in the Liouville sense and has the involutive system of conserved $F_{m}$ :

$$
\begin{aligned}
& F_{m}=\frac{1}{2}\left(<\Lambda^{m+1} p, p>-<\Lambda^{m+1} q, q>\right)-\frac{1}{2}<p, p><\Lambda^{m} q, q>+ \\
& \frac{1}{2}<q, q><\Lambda^{m} q, q>+\frac{1}{2} \sum_{i+j=m}\left|\begin{array}{cc}
<\Lambda^{i} q, q> & <\Lambda^{i} q, p> \\
<\Lambda^{j} p, q> & <\Lambda^{j} p, p>
\end{array}\right|, \quad m=0,1,2, \ldots
\end{aligned}
$$

Note $F_{0}=H$.

Consider the restriction of the integrable Hamiltonian system (39) on the following symplectic submanifold $M^{2 N-2}$

$$
M^{2 N-2}=\left\{(p, q) \in R^{2 N} \mid F \equiv \frac{1}{2}\left(<\Lambda^{-1} q, q>-1\right)=0, G \equiv<\Lambda^{-1} p, q>=0\right\} .
$$

On $M^{2 N-2}$, the Hamiltonian $H^{*}$ should be chosen as

$$
\begin{aligned}
& H^{*}=H-\lambda F-\mu G, \\
& \mu=\left.\frac{(H, F)}{(G, F)}\right|_{M^{2 N-2}}=0, \quad \lambda=\left.\frac{(H, G)}{(F, G)}\right|_{M^{2 N-2}}=\frac{<q, q>-<p, p>}{<\Lambda^{-2} q, q>} .
\end{aligned}
$$

Proposition 9 The Hamiltonian system $\left(H^{*}\right)$ on $M^{2 N-2}$ defined by (41)

$$
\left(H^{*}\right):\left\{\begin{array}{l}
q_{x}=\frac{\partial H^{*}}{\partial p}=\Lambda p-<p, q>q \\
p_{x}=-\frac{\partial H^{*}}{\partial p}=\Lambda q-2<q, q>q+<p, q>p+\frac{<q, q>-<p, p>)}{2<\Lambda^{-2} q, q>} \Lambda^{-1} q
\end{array}\right.
$$

is integrable and possesses the involutive system $F_{m}^{*}$

$$
F_{m}^{*}=F_{m}-\lambda_{m} F
$$

with

$$
\lambda_{m}=\left.\frac{\left(F_{m}, G\right)}{(F, G)}\right|_{M^{2 N-2}}=\frac{<\Lambda^{m-1} q, q>(<q, q>-<p, p>)}{<\Lambda^{-2} q, q>} .
$$


In (43), set

$$
\left\{\begin{array}{l}
P=\frac{1}{2}(q+p) \\
Q=\frac{1}{2} \varepsilon(q-p), \quad \varepsilon= \pm 1
\end{array}\right.
$$

and

$$
\left\{\begin{array}{l}
u=-\varepsilon<p, q> \\
v=\frac{1}{2} \varepsilon \frac{<q, q>-<p, p>}{<\Lambda^{-2} q, q>} \\
w=-\varepsilon<q, q>
\end{array}\right.
$$

then (43) is equivalent to

$$
\left\{\begin{array}{l}
Q_{j, x}=-\left(\lambda_{j}+\varepsilon w+\varepsilon \lambda_{j}^{-1} v\right) Q_{j}+\left(u-w-\lambda_{j}^{-1} v\right) P_{j} \\
P_{j, x}=\left(u+w+\lambda_{j}^{-1} v\right) Q_{j}+\left(\lambda_{j}+\varepsilon w+\varepsilon \lambda_{j}^{-1} v\right) P_{j}
\end{array} \quad j=1,2, \ldots, N\right.
$$

which is exactly a new eigenvalue problem

$$
y_{x}=\left(\begin{array}{rr}
-\lambda-\varepsilon w-\varepsilon \lambda^{-1} v & u-w-\lambda^{-1} v \\
u+w+\lambda^{-1} v & \lambda+\varepsilon w+\varepsilon \lambda^{-1} v
\end{array}\right) y \quad \varepsilon= \pm 1
$$

with $y=\left(Q_{j}, P_{j}\right)^{T}, \lambda=\lambda_{j}$.

It is easy to get the spectral gradient $\nabla \lambda_{j}$ with regard to the potential $u, v$ and $w$ of (49)

$$
\nabla \lambda_{j}=\left(\begin{array}{c}
\delta \lambda_{j} / \delta u \\
\delta \lambda_{j} / \delta v \\
\delta \lambda_{j} / \delta w
\end{array}\right)=\left(\begin{array}{r}
-Q_{j}^{2}+P_{j}^{2} \\
-\lambda_{j}^{-1}\left(P_{j}+\varepsilon Q_{j}\right)^{2} \\
-\left(P_{j}+\varepsilon Q_{j}\right)^{2}
\end{array}\right), \quad j=1,2, \ldots, N
$$

Introduce the C. Neumann constraint condition

$$
\left(\begin{array}{c}
-u \\
-\varepsilon \\
w
\end{array}\right)=\sum_{j=1}^{N} \varepsilon \cdot \nabla \lambda_{j}
$$

which directly yields the first and the third equalities of (47) and

$$
<\Lambda^{-1}(P+\varepsilon Q), P+\varepsilon Q>=1, \quad \text { i.e. } \quad<\Lambda^{-1} q, q>=1 .
$$

After the $x$-derivative is made in the relation $\left\langle\Lambda^{-1} p, q>=0\right.$, i.e., $<\Lambda^{-1}(P-\varepsilon Q), P+$ $\varepsilon Q>=0$, and (48), (51) are used, the second equality of (47) is promptly obtained. Thus, (47) is actually produced by the C. Neumann constraint (51) and $\left\langle\Lambda^{-1} q, p\right\rangle=0$. So is the symplectic submanifold $M^{2 N-2}$ defined by (41).

Proposition 10 The symplectic submanifold $\left(M^{2 N-2},\left.d p \wedge d q\right|_{M^{2 N-2}}\right)$ defined by (41) in the symplectic space $\left(R^{2 N-2}, d p \wedge d q\right)$ is generated by the C. Neumann constraint (51), and the completely integrable Hamiltonian system (43) is actually the generalized C. Neumann system of the new eigenvalue problem (49). 
Conclusion. Through some examples lined above, it appears that the scope of completely integrable systems in the Liouville sense is further enlarged. We can also apply this approach to other completely integrable Hamiltonian systems, and produce other new generalized C. Neumann integrable systems.

\section{Acknowledgements}

The author wishes to thank Professor $\mathrm{Cu}$ Chaohao and Professor $\mathrm{Hu}$ Hesheng for their enthusiastic guidance.

\section{References}

[1] Cao C.W., Sci. China A,, 1990, V.33, 528.

[2] Cao C.W. and Geng X.G., Classical integrable systems generated through nonlinearization of eigenvalue problems, in: Research Reports in Physics, Nonlinear Physics, eds. C. Gu, Y. Li and G. Tu, Berlin, Springer, p.68.

[3] Konopelchenko B., Sidorenko J. and Strampp W., Phys. Lett. A, 1991, V.157, 17; Cheng Y. and Li Y.S., Phys. Lett. A, V.157, 22.

[4] Cao C.W., Acta Math. Sin. New Series, 1990, V.6, 35;

Cao C.W. and Geng X.G., J. Phys. A: Math. Gen., 1990, V.23, 4117; J. Math. Phys., 1991, V.32, 2323.

[5] Xu T.X. and Mu W.H., Phys. Lett. A, 1990, V.147, 125;

Gu Z.Q., it Chin. Sci. Bull., 1991, V.36, 1683;

Geng X.G., Physica A, 1992, V.180, 241; Phys. Lett. A, 1992, V.162, 375;

Qiao Z.J., Phys. Lett. A, 1993, V.172, 224; J. Math. Phys., 1993, V.34, 3110; J. Phys. A: Math. Gen., 1993, V.26, 4407.

[6] Antonowicz M. and Wojciechowski S., Phys. Lett. A, 1990, V.147, 455; J. Phys. A: Math. Gen., 1991, V.24, 5043; J. Math. Phys., 1992, V.33, 2115.

[7] Xu B. and Li Y.S., J. Pys. A: Math. Gen., 1992, V.25, 2957;

Blaszak M., Phys. Lett. A, 1993, V.174, 85.

[8] Zeng Y.B., Chen D.Y. and Li Y.S., Chin. Ann. Math. B, 1992, V.13, 327.

[9] Qiao Z.J., J. of Math.. Res. and Exp., 1993, V.13, 377.

[10] Qiao Z.J., A completely integrable C. Neumann system associated with the Levi hierarchy, preprint.

[11] Moser J., Integrable Hamiltonian system and spectral Theory, in: Proc. of 1983 Beijing Symp. on Diff. Geom. and Diff. Eq's, Science Press, Beijing, 1986, p.157.

[12] Arnol'd V.I., Mathematical Methods of Classical Mechanics, Berlin, Shpringer, 1978.

[13] Li Y.S. and Zhuang D.W., Scientia Sinica A,, 1983, V.13, 107.

[14] Hu X.B., Northeastern Math. J., 1990, V.6, 187.

[15] Eilbeck J.C., Enol'ski V.Z., Kuznetsov V.B., Leykin D.V., Classical structure for a hierarchy of onedimensional particle systems separable in parabolic coordinates, J. Nonlin. Math. Phys., 1994, V.1, N 3, 275-294. 